able, for a man to be so indifferent to the worst that circumstance or human malice can do, that in effect he becomes the master of circumstance and malice is disarmed against him. Spinoza is also the only philosopher known to history who earned an honest living by the work of his hands. A. D. RitchiE

\section{ADVANCED THEORY OF STATISTICS}

\section{The Advanced Theory of Statistics}

By Maurice G. Kendall. Vol. 2. Pp. vii+521. (London: Charles Griffin and Co., Ltd., 1946.) 50s. net.

7

HE first volume of this work, reviewed by Prof. R. A. Fisher in Nature of October 16, 1943, covered the subjects of frequency distributions and their properties; theories of probability and sampling and the sampling distributions to which they lead; and the theory of correlation. Chapter 12 on the $\chi^{2}$ distribution was described by the author as something of an intrusion. A new edition of volume 1 in 1945 had no alterations of substance. The present very welcome volume 2 falls into five sections, dealing with estimation, the theory of statistical tests including the analysis of variance, regression analysis, the design of statistical inquiries, and the analysis of time series.

Prof. A. D. Ritchie, in Nature of November 2, 1946 , p. 639 , has drawn a distinction between the man of science and the technologist which can, I think, be applied, with a difference, to mathematicians. Some, if they carry out calculations, do so as practice for the sake of understanding. Others, though they must understand first, do so for the sake of the practical application. I may perhaps speak for those who want to apply statistical methods in various fields - in my case experimental psychology and education-but want also to understand them, indeed become interested in them for their own sake. Such must welcome Mr. Kendall's book warmly, and perhaps especially this second volume, in which Chapters 23 and 24 on the analysis of variance, and 25 on the design of sampling inquiries, are of peculiar interest to them.

In agricultural experiments, properly designed, there seldom arises the problem of "unequal numbers in subclasses" (par. 24.6 et seq.). But in all sociological experiments, numbers in subclasses are liable to inequality. Absences from school affect one class more than another. An island population does not fall into occupational or racial classes of equal size. So the procedures to be adopted with 'nonorthogonal' data (pp. 220-9) are of importance. I am sorry there is only a page and a half (pp. 262-3) about 'confounding'. It is very useful, however, to have everything in one book, and the examples and exercises (easy and difficult) are an admirable training and clarification.

Mr. Kendall describes the work on canonical correlations of Hotelling and others, on pp. 348-58. Their perusal reminded me of a problem which I posed a few years ago, to which $I$ have not received an answer. In work with intelligence tests the canonical correlation technique appears as the problem of finding sets of weights to apply to two batteries of tests so that the correlation coefficient of the two weighted scores is a maximum. I applied this in 1940 (Brit. J. Psychol., 30, 358) to the problem of finding what weights would maximize a battery's reliability, that is, its correlation with a duplicate battery. These weights will, however, be different from those which cause this battery to give the best prediction of some 'criterion', such as future success in a secondary school, and straining after reliability may spoil prediction, and vice versa. The problem was to find weights which would make reliability equal to prediction and both as large as possible under this condition. In simple cases a solution can be found. But what is the general solution?

Students who are not primarily mathematicians very much need and welcome preliminary general explanations of the logic and purpose of the procedures to be proved and used in the succeeding pages, and some of them find geometrical analogies helpful. Given such assistance, they are able to follow the algebraic work more easily, or at least to appreciate its general trend, and to feel able to accept, not quite blindly, the formulæ arrived at.

Such assistance is not wanting in Mr. Kendall's volume - I may instance pp. 269-72 with the diagram, at the outset of the discussion of the general theory of significance tests, and the diagrams of confidence belts on pages 68,69 and 77 . In the latter I suggest the addition in dotted lines of the whole sausage-shaped region of acceptance, of which the shaded area is a section.

The theory of probability has always been an arena for controversies and even quarrels. Mr. Kendall has refused to take sides in present-day disputes, but has objectively described both points of view. About the different approaches to the treatment of confidence intervals and fiducial inference, differences which so easily escape from one's mind even when one has again and again grasped them fleetingly, he has written two admirably lucid chapters, and has had them read by Prof. Egon Pearson and Prof. R. A. Fisher respectively to ensure that he has at least not misrepresented them. To the outsider, now one and now the other view seems the better. In sociological work, it is important not merely to consider the possibility of a hypothesis being the true explanation, but also its relative standing when compared with alternatives. Neyman and Pearson make just this point, and in addition to defining regions within which we reject a hypothesis because the odds are so and so that the data did not arise from it (of which regions there may be many) they choose among them that region which minimizes the chance of accepting the hypothesis when it is false.

On the other hand, the Behren's test does not hold in the theory of confidence intervals, and the reply that "some questions may not have an answer" seems scarcely sufficient. Then Kendall protests that we can never test a fiducial distribution by drawing samples. But we can set up some apparatus corresponding to the value of $\theta$ and find experimentally the proportion of samples which give rise to $t$. Then we can change $\theta$ and repeat; and so on. Surely this is an experimental frequency distribution.

Many will look forward to the future publication which Mr. Kendall half promises, in which he hopes to show that all these disagreements are more apparent than real.

The book is beautifully printed and the proofreading has been excellent.

GODFREY ThOMSON 